\title{
Synthesis of Carmegliptin
}
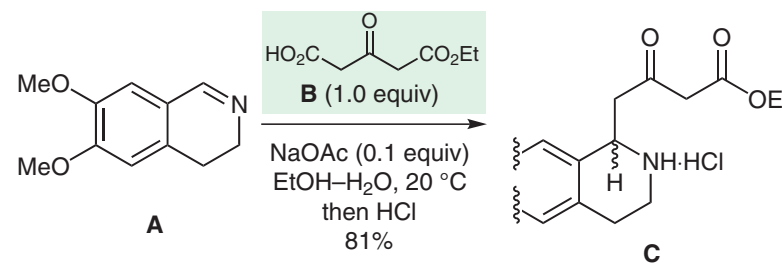
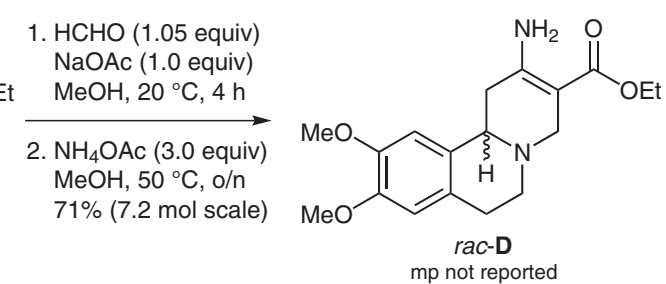

(S,S)-DBTA (1.06 equiv) $\mathrm{EtOH}, 60^{\circ} \mathrm{C}, 24 \mathrm{~h}$ $93 \%(74.5 \mathrm{~mol}$ scale $)$ crystallization-induced

1. $\mathrm{NaBH}_{4}$ (1.35 equiv) $\mathrm{Phl}(\mathrm{OAc})_{2}$ (1.1 equiv) $\mathrm{KOH}$ (5.0 equiv) $\mathrm{MeCN}-\mathrm{H}_{2} \mathrm{O}, 28^{\circ} \mathrm{C}, 4 \mathrm{~h}$ $85 \%$ (296 mol scale)

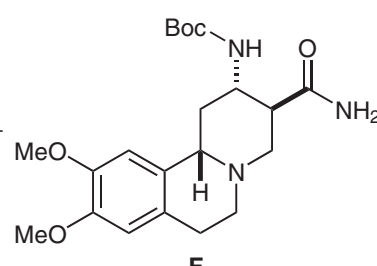

$\mathrm{mp}$ not reported<smiles>COc1cc2c(cc1OC)[C@@H]1C[C@H](NC(=O)OC(C)(C)C)C(N)CN1CC2</smiles>
mp not reported<smiles>COc1cc2c(cc1OC)[C@]1(C)C[C@H](N)[C@@H](N3CC(CF)CC3=O)CN1CC2</smiles>

aq $\mathrm{HC}$

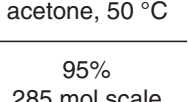

$285 \mathrm{~mol}$ scale TFA (5.0 equiv) $\mathrm{THF},-10$ to $0^{\circ} \mathrm{C}$

2. $\mathrm{Boc}_{2} \mathrm{O}$ (1.3 equiv)

$\mathrm{PhMe}-\mathrm{H}_{2} \mathrm{O}, 5^{\circ} \mathrm{C}$ to r.t.

3. $\mathrm{NaOMe}$ (3.0 equiv)

$\mathrm{HCONH}_{2}$ (10 equiv)

$\mathrm{THF}, 35^{\circ} \mathrm{C}$

$85 \%$ (290 mol scale)

dynamic resolution

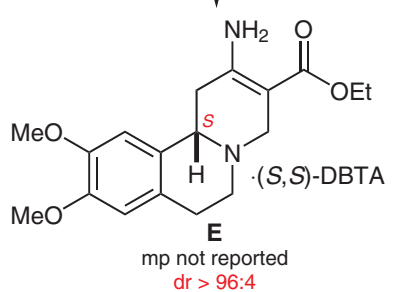

$d r>96: 4$

DBTA = dibenzoyltartaric acid<smiles>COc1cc2c(cc1OC)C1(CC2)NCCN2CC(NC(=O)CC(CO)CF)[C@H](NC(=O)OC(C)(C)C)CC21</smiles>

$78 \%$ from G $1 . \mathrm{MsCl}^{\mathrm{Et}} \mathrm{Et}_{3} \mathrm{~N}, \mathrm{THF}, 22^{\circ} \mathrm{C}$ 2. LHMDS, THF, -10 to $0^{\circ} \mathrm{C}$<smiles>COc1cc2c(cc1OC)[C@@H]1C[C@H](NC(=O)OCc3ccccc3)C(N3CC(CF)CC3=O)CN1CC2</smiles>

\section{Key words}

\section{carmegliptin}

DPP-4 inhibitors

Mannich reaction

crystallizationinduced dynamic resolution

Hofmann rearrangement

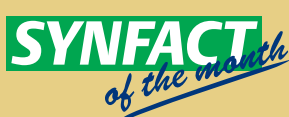

Significance: Carmegliptin is a dipeptidyl dipeptidase-IV inhibitor under development for the treatment of type-2 diabetes. Over $1000 \mathrm{~kg}$ was synthesized by the route depicted featuring an efficient crystallization-induced dynamic resolution of rac-D and a large-scale Hofmann rearrangement of the amide $\mathbf{F}$.
Comment: J.-M. Adam et al. (Org. Process Res. Dev. 2011, 15, 515) describe the synthesis of lactone $\mathbf{H}$ by asymmetric hydrogenation $(10 \mathrm{~kg}$ scale), but a route starting from commercial (S)tert-butyl glycidyl ether (derived from a Jacobsen hydrolytic kinetic resolution) delivered $>500 \mathrm{~kg}$ of H.

SYNFACTS Contributors: Philip Kocienski

Synfacts 2011, 8, 0811-0811 Published online: 20.07.2011

DoI: 10.1055/s-0030-1260677; Reg-No.: K03611SF 